\title{
Polygon "Simeiz-Katsively" - study of changes in the geodynamic, heliogeophysical and climatic characteristics of Crimea
}

\author{
Alexander Volvach ${ }^{1, *}$, Galina Kurbasova ${ }^{1}$, Andrej Dmytrotsa ${ }^{1}$, Dmitry Neyachenko ${ }^{1}$, \\ Konstantin Kanonidi ${ }^{2}$, and Olga Boborykina ${ }^{3}$ \\ ${ }^{1}$ Radio Astronomy and Geodynamics Department, Crimean Astrophysical Observatory RAS, \\ Katsively \\ ${ }^{2}$ Pushkov Institute of Terrestrial Magnetism, Ionosphere and Radio Wave Propagation RAS, Moscow \\ ${ }^{3}$ Schmidt Institute of Physics of the Earth RAS, Moscow
}

\begin{abstract}
The solution to the problem of forecasting catastrophic natural and technological phenomena in Crimea is associated with the organization of complex observations in a network of local geodynamic polygons. Currently conducted on-site observations involved in the solution of problems of geodynamic and environmental monitoring in international cooperation. To study lithospheric deformation, the reasons for their cause, the search for prognostic indicators, preparing regional seismic events of natural and anthropogenic origin in the Crimean region, in 2018, geodynamic testing area "Simeiz-Katsively" have been expanded tiltmeter and magnetovariational stations, included in the national observation network.
\end{abstract}

\section{Introduction}

To explore emerging and developing deformation processes, movements of lithospheric plates are created geodynamic polygons, experimental equipment, which implements the comprehensive observational techniques of radio interferometry with very long baseline (VLBI), the laser ranging to artificial satellites (LRS) and the satellite systems GPS/GLONASS.

In Crimea, established and partially equipped with necessary equipment for the integrated monitoring of geodynamic polygon "Simeiz-Katsively". Currently conducted on-site observations involved in the solution of problems of geodynamic and environmental monitoring in international cooperation.

To study lithospheric deformation, the reasons for their cause, the search for prognostic indicators, preparing regional seismic events of natural and anthropogenic origin in the Crimean region, in 2018, geodynamic testing area «Simeiz-Katsively" have been expanded tiltmeter and magnetovariational stations, included in the national observation network.

\footnotetext{
*Corresponding author: volvach@bk.ru
} 


\section{Experimental base for geodynamic observations}

Created in Crimea geodynamic site "Simeiz-Katsively" have formed the basis of ground and space-based time series of observations of climatic and geophysical characteristics of the Crimea, especially the Mountainous parts (Fig. 1).

Applied a fundamentally new approach - geodynamic site «Simeiz-Katsively” consists of three mutually supportive of each other technology observations: VLBI, laser ranging satellites and global navigation satellite system [1,2]. According geodynamic observation stations of the polygon is defined with an accuracy of few millimeters in the horizontal and vertical component of speed of movement of the Crimean Peninsula and the Eurasian tectonic plates. To ensure monitoring of large-scale tectonic processes and control of "fast" deformations in local seismic and landslide hazardous zones of the Crimean peninsula, the local speed and direction of movement of the coast of the Southern Crimean coast near the settlements of Simeiz-Katsively were determined by geodynamic measurements [3].

The analysis of climatic characteristics of the Peninsula of Crimea held on average daily and average annual satellite measurements of climatic parameters gave the possibility to obtain information on insolation and warming of the earth's surface in the Crimea, the surface temperature on the Peninsula as a whole, and the abnormal lowering of the temperature of the earth's surface in the extreme Eastern point of the Peninsula [4].

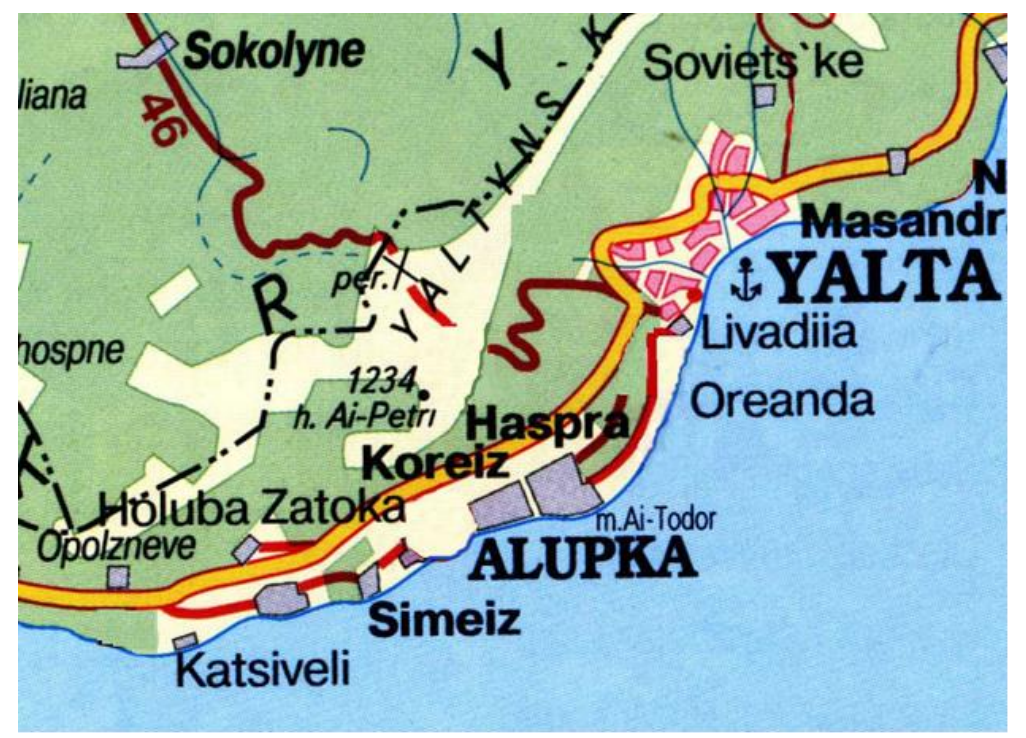

Fig. 1. The "Simeiz-Katsively" test area.

The positions of the points in the "Simeiz-Katsively" test area have been determined by special GPS survey campaign. Results are presented in Table 1 and 2.

Table 1. Final solution for coordinates of points in the Simeiz-Katsively area.

\begin{tabular}{|c|c|c|c|}
\hline Station & $\mathrm{X}, \mathrm{m}$ & $\mathrm{Y}, \mathrm{m}$ & $\mathrm{Z}, \mathrm{m}$ \\
\hline RT22G & 3785230.904 & 2551207.524 & 4439796.448 \\
\hline RT22 & 3785223.388 & 2551202.451 & 4439787.574 \\
\hline KATS-SLR & 3785944.414 & 2550780.660 & 4439461.335 \\
\hline SIMI-SLR & 3783902.266 & 2551405.032 & 4441257.506 \\
\hline GPS-CRAO & 3783897.116 & 2551404.411 & 4441264.266 \\
\hline
\end{tabular}


Table 2. Offsets for 10 years of the coordinates of points in the Simeiz-Katsively area.

\begin{tabular}{|c|c|c|c|}
\hline Station & $\mathrm{dX}$ & $\mathrm{dY}$ & $\mathrm{dZ}$ \\
\hline RT22G & -0.216 & 0.126 & 0.077 \\
\hline KATS-SLR & -0.200 & 0.137 & 0.032 \\
\hline SIMI-SLR & -0.230 & 0.134 & 0.069 \\
\hline
\end{tabular}

Tiltmeter station, installed in Katsively on the territory of the Crimean astrophysical observatory, designed and manufactured by D.G. Gridnev in collaboration with employees of IZMIRAN. It is composed of two identical quartz tiltmeter, which are located on the plane with mutually perpendicular arrangement of the axes of rotation of pendulums. The design of the tiltmeter shown in Fig. 2.

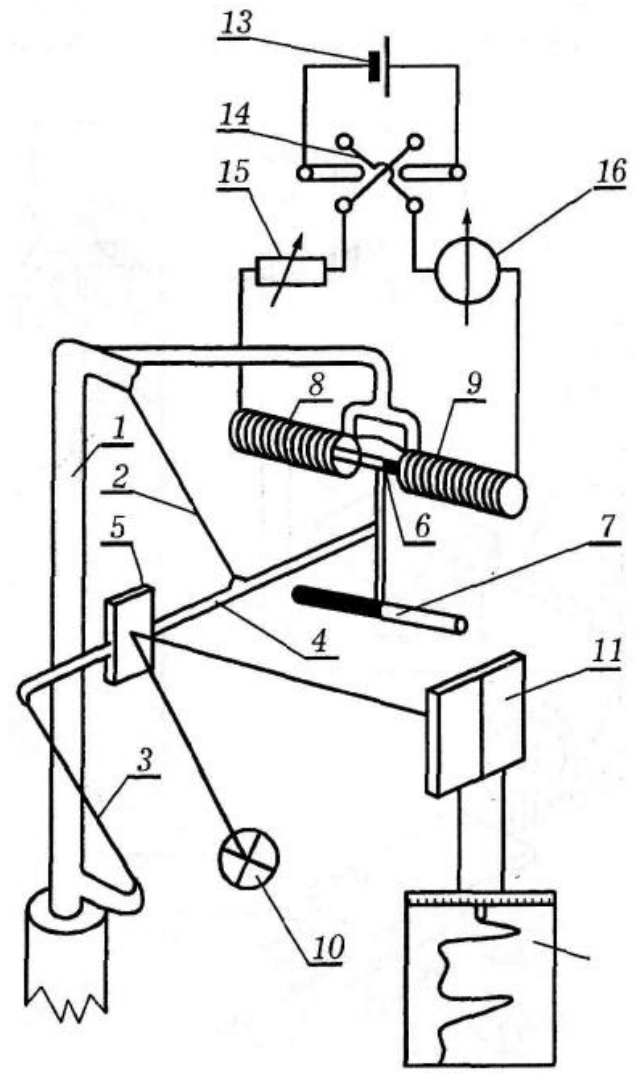

Fig. 2. Tiltmeter with control device and calibration using electromagnetic force.

In the mounting frame 1, a horizontal pendulum 4 is suspended on elastic threads 2,3 with a mirror 5 and two identical rod, antiparallel permanent magnets 6 , 7, rigidly fixed horizontally at the end of the pendulum 4 perpendicular to its axis in one vertical plane at a distance of no more than $10 \mathrm{~mm}$ from each other from friend. The ends of the magnet 6 are placed in the solenoids 8,9 , rigidly fastened to the mounting frame 1 so that when the circuit is open, they coincide with the centers of the solenoids, and the axis of the magnet coincides with the axis of the solenoids. The tiltmeter contains a photoelectric converter, consisting of a illuminator 10, a mirror 5 and a differential photocell 11 connected to the recorder 12 . Solenoids 8,9 are connected to a control unit consisting of a power source 13 , a polarity switch 14, a resistance store 15 , and a mirror calcareous microammeter 16 . 
The tiltmeter does not contain current leads to the sensitive system; therefore, the possibility of drift of the null point is excluded and linearity of the instrument readings is ensured. The distance between the magnets does not exceed $10 \mathrm{~mm}$, which allows to eliminate not only the effects of changes in the external magnetic field, but also changes the gradients of the external magnetic field. The placement of the ends of the magnet in the middle of the solenoids provides a linear relationship between the magnitude of the deviation of the pendulum and the magnitude of the current in the circuit of the solenoids. These tiltmeters have found wide application in our country, and also operated in Belgium and Luxembourg.

\section{Conclusion}

To study lithospheric deformation, the reasons for their cause, the search for prognostic indicators, preparing regional seismic events of natural and anthropogenic origin in the Crimean region, in 2018, geodynamic testing area «Simeiz-Katsively" have been expanded tiltmeter and magnetovariational stations, included in the national observation network.

\section{References}

1. A. Volvach, L.Petrov, N. Nesterov, Kinematics and Physics of Celestial Bodies. Supplement Series, 1, 116 (1999)

2. N. Nesterov, A. Volvach, International VLBI service for geodesy and astrometry. Goddard Space Flight Center. Greenbelt, MD 20771 USA. P.96-100 (1999)

3. L. Petrov, A. Volvach, N. Nesterov, Kinematics and Physics of Celestial Bodies, 17, 5, 424 (2001)

4. A.E. Volvach, G.S. Kurbasova, Geofizicheskiy Zhurnal-Geophysical Journal, 41, 1, 192 (2019) 